\title{
Glucosylglycerol on performance of prebiotic potential
}

\section{Thornthan Sawangwan}

Department of Biotechnology, Faculty of Science, Ramkhamhaeng University, Huamark, Bangkapi, Bangkok 10240, Thailand

Corresponding Author: Thornthan Sawangwan, $\mathrm{PhD}$, Department of Biotechnology, Faculty of Science, Ramkhamhaeng University, Huamark, Bangkapi, Bangkok 10240, Thailand

Submission Date: October 9, 2015, Acceptance date: December 25, 2015: Publication date: December 29, 2015

\begin{abstract}
:
Background: Glucosylglycerol (GG), an organic compound constitutes a structurally diverse group of small organic molecules, which has a glycosidic chemical structure, a powerful osmolyte which is catalyzed by sucrose phosphorylase in the presence of sucrose and glycerol as a donor and acceptor substrate.
\end{abstract}

Context and purpose of this study: This research aims to study the potential of prebiotic properties from GG compared with three commercial oligosaccharides (FOS, inulin and lactulose) which also have prebiotic properties.

Results: The cultures with GG and FOS showed statistically significant difference $(\mathrm{P}<0.05)$ capacity to stimulated both probiotic strains (Lactobacillus acidophilus and Lactobacillus plantarum) and presented high ability to inhibit four intestinal pathogens (Bacillus cereus, Escherichia coli, Samonella paratyphi and Staphyloccous aureus), as displayed from wider inhibition zone compared with the culture without oligosaccharide. The tolerance ability of different gastrointestinal conditions (alpha-amylase, bile extract and $\mathrm{HCl}$ ) in the culture medium with GG presented the highest percentage of survival (6.50\%) after being incubated with $\mathrm{HCl}$ for 3 hours (significant difference as $\mathrm{P}<0.05$ ). The culture of $L$. acidophilus with $\mathrm{GG}$ in the medium displayed maximum lactic acid concentration $(1.46 \mathrm{mg} / \mathrm{mL})$ after 48 incubation hours.

Conclusions: GG has high potential of prebiotic properties for probiotic growth stimulation, pathogenic inhibition and gastrointestinal tolerance. Additionally, GG can introduce probiotic for high production of lactic acid concentration in the culture media. From these results, GG is promising for application and development to become an effective functional food in the coming future. 
Keywords: glucosylglycerol, prebiotic, probiotic, pathogen inhibition, gastrointestinal tolerance, lactic acid

\section{INTRODUCTION:}

Today, consumer dietary trends not only include clean foods and good taste, but also a preference for functional foods which have high nutrition, positive effect on health, and reduce risk of disease. Among functional foods, prebiotics are extensively popular for consumer. There are many studies which cite the advantages of prebiotic foods. For example, there is the growth stimulation of probiotic $[1,2]$, inhibition of intestinal pathogen [3, 4], and promote tolerance of probiotic in gastrointestinal condition [5].

Glucosylglycerol (GG), is a compatible compound which has a glycosidic bond and hydroxyl groups in its chemical structure (Figure 1). GG is synthesized via biocatalytic process of recombinant sucrose phosphorylase from Leuconostoc mesenteroides with sucrose and glycerol as substrate [6]. GG serves as an essential function in protecting cell and protein against extremely conditions (high salt concentration, different temperature and other forms of external stress). Based on the chemical structure of oligosaccharides in Figure 2 (fructo-oligosaccharide; FOS, inulin and lactulose), they also all have glycosidic bonds and hydroxyl groups in their structure. Moreover, these oligosaccharides are well documented in the positive effects of probiotic and prebiotic potential [7-10].

In this study, GG was demonstrated to have prebiotic properties (probiotic growth stimulation, intestinal pathogen inhibition and gastrointestinal tolerance) compared with 3 commercial oligosaccharides (FOS, inulin and lactulose). Additionally, lactic acid production from the cultures with all oligosaccharides were analyzed by high performance liquid chromatography; HPLC.

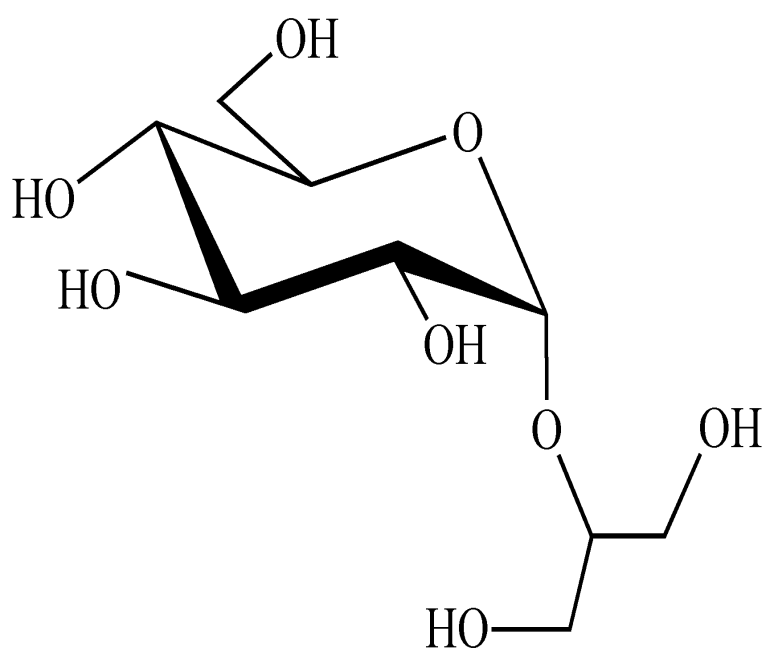

Figure 1. Glucosylglycerol (GG) chemical structure 

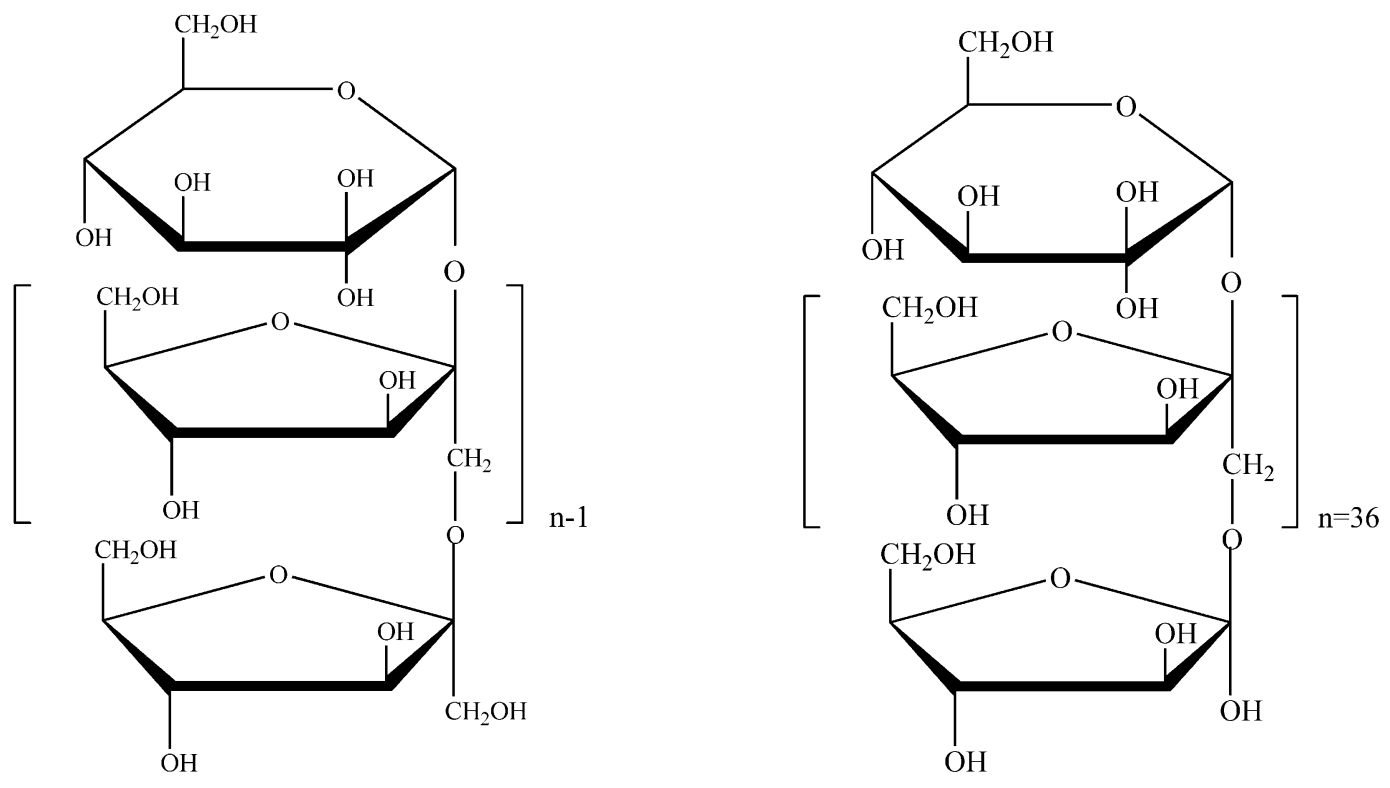

(A)

(B)

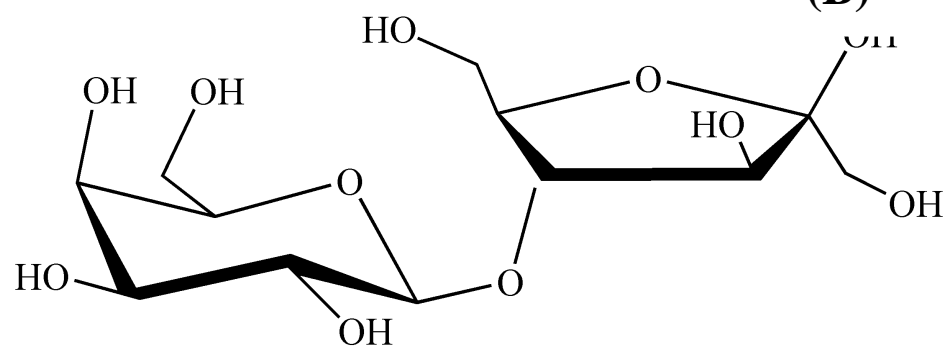

(C)

Figure 2. Chemical structure of commercial oligosaacharides with prebiotic properties; FOS (A), inulin (B) and lactulose (C)

\section{MATERIALS AND METHODS:}

\section{Materials}

GG was kindly provided from the Institute of Biotechnology and Biochemical Engineering, Graz University of Technology, Graz, Austria. Fructo-oligosaccharide (FOS), inulin and lactulose are commercial oligosaccharides used as comparable for prebiotic properties. All other chemicals used were analytical grade.

Probiotic strains (L. acidophilus TISTR 1338 and L. plantarum TISTR 1465) were kindly provided from the Thailand Institute of Scientific and Technological Research. Intestinal pathogens (Bacillus cereus, Escherichia coli, Salmonella paratyphi and Staphylococcus aureus) were obtained from Department of Biology, Faculty of Science, Ramkhamhaeng University. All other chemicals used were analytical grade.

\section{Probiotic growth stimulation}

L. acidophilus and L. plantarum were grown at $37^{\circ} \mathrm{C}$ for 48 hours under anaerobic condition in Man Rogosa Sharpe; MRS broth (used as control) and compared with the culture medium 
supplemented with $1 \mathrm{mg} / \mathrm{ml}$ of each FOS, GG, inulin and lactulose. After incubation, the cultures were monitored by measuring an optical cell density with a spectrophotometer at $620 \mathrm{~nm}[11$, 12].

\section{Pathogenic inhibition}

The cultivations of L. acidophilus and L. plantarum at $37^{\circ} \mathrm{C}$ for 48 hours in MRS broth complemented with $1 \mathrm{mg} / \mathrm{ml}$ of each oligosaccharide were centrifuged at 8,000 rpm (revolutions per minute), at $4^{\circ} \mathrm{C}$ for 15 minutes. Supernatant was separately collected. Pathogenic strains (B. cereus, E. coli, Salmonella paratyphi and Staphylococcus aureus) were cultivated in nutrient broth; NB at $37^{\circ} \mathrm{C}$ for 24 hours. Aliquot of pathogenic cultivation $\quad(50 \mu \mathrm{l})$ was inoculated in nutrient agar; NA by spread plate technique and dried. Supernatant from each probiotic culture was dropped onto the sterilized disks of the filter papers and placed on the plates as prepared above. After incubation at $37^{\circ} \mathrm{C}$ for 24 hours, inhibition efficiency was illustrated by diameter of the clear zone from the probiotic supernatant and compared with the clear zone from the culture media without oligosaccharide as a control. [12]

\section{Gastrointestinal tolerance}

The cell pellets from previous preparation of L. acidophilus and L. plantarum were washed twice by using $1 \mathrm{ml}$ of phosphate buffer saline; PBS buffer $\mathrm{pH} 7$ and centrifuged at $8,000 \mathrm{rpm}, 4^{\circ} \mathrm{C}$ for 15 minutes. Then they were incubated in MRS broth with $1 \mathrm{ml}$ of each gastrointestinal conditions (100 Unit/ml alpha-amylase, $0.3 \% \mathrm{w} / \mathrm{v}$ bile extract and $0.1 \mathrm{M} \mathrm{HCl})$ at $37^{\circ} \mathrm{C}$ for $0.5,1$, 2 and 3 hours. Gastrointestinal tolerance was determined by the percentage of CFU/ml (colony forming unit) from Lactobacillus survival using the spread plate technique, and were incubated at $37^{\circ} \mathrm{C}$ for 48 hours with triplicate experiments [13].

\section{Lactic acid formation}

The probiotic medium cultures supplemented of each oligosaccharide at 24 and 48 incubation hours were analyzed lactic acid concentration by HPLC using a column of Dionex Ion-Pac ICEAS6, $259 \times 9 \mathrm{~mm}$ at $90^{\circ} \mathrm{C} .4 \mathrm{mM}$ of heptafluorobutyric acid and $5 \mathrm{mM}$ of tetrabutylammoniumhydroxide were used as the mobile phases at a constant flow rate of $1 \mathrm{ml} / \mathrm{minute}$. Lactic acid standard was used for calculation of lactic acid identification and quantification [14].

\section{RESULTS AND DISCUSSION:}

Probiotic strains (L. acidophilus and L. plantarum) were grown under anaerobic condition in the culture medium supplemented of each oligosaccharide (FOS, GG, inulin and lactulose).

\section{Probiotic growth stimulation}

After incubation, the growth of probiotics (L. acidophilus and L. plantarum) were observed by monitoring the optical cell density in spectrophotometer at $620 \mathrm{~nm}$. The results are presented in Table 1. All cultures show no significant difference to stimulate growth of both probiotic strains. Noticeably, in the culture of L. plantarum, all oligosaccharides showed higher cell density compared to the control culture (without oligosaccharide). It has also been documented in [15] 
commercial oligosaccharides, such as FOS, GOS and inulin for growth stimulation of Lactobacillus strains (L. acidophislus and L. plantarum) and Bifidobacteria strains (B. adolescentis, B. breve, B. infantis and B. longum).

Table 1. The optical cell density of probiotic strains (L. acidophilus and L. plantarum) cultivation at $37^{\circ} \mathrm{C}$ for 48 hours under anaerobic condition in media culture with oligosaccharides supplement

\begin{tabular}{|ccc|}
\hline \multirow{2}{*}{ Sample } & \multicolumn{2}{c|}{ OD620 nm } \\
\cline { 2 - 3 } & L. acidophilus & L. plantarum \\
\hline control & $1.540 \pm 0.013$ & $1.543 \pm 0.002$ \\
FOS & $1.521 \pm 0.005$ & $1.579 \pm 0.001$ \\
GG & $1.512 \pm 0.011$ & $1.563 \pm 0.001$ \\
inulin & $1.518 \pm 0.001$ & $1.567 \pm 0.004$ \\
lactulose & $1.512 \pm 0.002$ & $1.611 \pm 0.001$ \\
\hline
\end{tabular}

\section{Pathogenic inhibition}

The results of the pathogenic inhibition efficiency show in Table 2, with the statistically significant difference at $\mathrm{P}<0.05$. Both of probiotic strains in medium complemented of FOS, GG, inulin and lactulose presented the inhibition zone (clear zone) of intestinal pathogens. In the culture of L. acidophilus with FOS and GG supplemented in the medium, there was a display of a wilder clear zone from inhibition of $B$. cereus for 0.90 and $0.98 \mathrm{~cm}$ and inhibition of Salmonella parathypi for 1.50 and $1.20 \mathrm{~cm}$. While, L. plantarum culture contained with GG in the medium presented high efficiency to $E$. coli inhibition with the clear zone of $1.35 \mathrm{~cm}$. Significantly, GG and FOS in the culture medium of both probiotic strains showed higher efficiency of all pathogenic inhibition than the other oligosaccharides. This phenomenon may be explained by how probiotic strains are preferable to utilized carbon source from GG and FOS in order to induce antimicrobial compound as lactic acid and peroxide for pathogenic inhibition. There is some evidence from [16] which describes Lactobacillus strains (L. plantarum K1, L. plantarum $\mathrm{O} 1$, L. helveticus $\mathrm{K}$ and L. casei $\mathrm{O}$ ) cultivated with FOS showed inhibition activity with B. subtilis, E. coli and Staphylococcus aureus. 
Table 2. The diameter of clear zone from probiotic strains (L. acidophilus and L. plantarum) inhibiting of intestinal pathogens (B. cereus, E. coli, Salmonella paratyphi and Staphylococcus aureus) in the culture with oligosaccharides

\begin{tabular}{|lllll|}
\hline \multirow{4}{*}{ Sample } & clear zone diameter $(\mathbf{c m})$ & & \\
\cline { 2 - 5 } & B. cereus & $\boldsymbol{E}$. coli & $\begin{array}{l}\text { Salmonella } \\
\text { paratyphi }\end{array}$ & $\begin{array}{l}\text { Staphylococcus } \\
\text { aureus }\end{array}$ \\
\hline L. acidophilus & & & & \\
Control & $0.75 \pm 0.071$ & $0.75 \pm 0.071$ & $1.05 \pm 0.071$ & $0.28 \pm 0.106$ \\
FOS & $0.90 \pm 0.035$ & $0.85 \pm 0.212$ & $1.50 \pm 0.283$ & $0.73 \pm 0.071$ \\
GG & $0.98 \pm 0.035$ & $1.05 \pm 0.212$ & $1.20 \pm 0.141$ & $0.83 \pm 0.177$ \\
inulin & $0.78 \pm 0.106$ & $0.85 \pm 0.071$ & $0.95 \pm 0.071$ & $0.33 \pm 0.035$ \\
lactulose & $0.53 \pm 0.106$ & $0.95 \pm 0.071$ & $0.75 \pm 0.071$ & $0.28 \pm 0.035$ \\
\hline L. plantarum & & & & \\
Control & $0.73 \pm 0.035$ & $0.88 \pm 0.177$ & $0.85 \pm 0.212$ & $0.80 \pm 0.000$ \\
FOS & $0.88 \pm 0.141$ & $0.80 \pm 0.283$ & $1.05 \pm 0.071$ & $0.78 \pm 0.035$ \\
GG & $0.98 \pm 0.035$ & $1.35 \pm 0.495$ & $1.05 \pm 0.212$ & $0.88 \pm 0.106$ \\
inulin & $0.70 \pm 0.071$ & $0.55 \pm 0.071$ & $0.85 \pm 0.071$ & $0.55 \pm 0.071$ \\
lactulose & $0.68 \pm 0.035$ & $0.78 \pm 0.035$ & $0.73 \pm 0.106$ & $0.68 \pm 0.035$ \\
\hline
\end{tabular}

\section{Gastrointestinal tolerance}

The tolerance of gastrointestinal conditions (alpha-amylase, bile extract and $\mathrm{HCl}$ ) were demonstrated by the survival of two probiotic strains (L. acidophilus and L. plantarum) after 0.53 hours in the medium supplement with FOS, GG, inulin and lactulose. Figure 3 shows the percentage survival of L. acidophilus (A) and L. plantarum (B) after incubation of alphaamylase, bile extract and $\mathrm{HCl}$. Interestingly after 3 hours, the culture with GG containing presented significantly higher survival percentage $(\mathrm{P}<0.05)$ of L. acidiophilus and L. plantarum in the conditions of alpha-amylase $(0.75 \%$ and $0.71 \%)$ and bile extract $(0.83 \%$ and $0.70 \%)$ than the other oligosaccharides. Significantly, both probiotic with all cultures were still grown in $\mathrm{HCl}$ condition even after 3 hours of incubation, especially L. acidophilus in culture with GG displayed highest percentage survival (6.50\%). However, both probiotic strains in the medium without any oligosaccharides (control) could not survive after 2 hours of all gastrointestinal conditions. This may be explained by a chemical structure of GG, which has many hydroxyl groups which have a good stabilizing property against extremely conditions as mentioned in [17]. Moreover, there are some reports [18, 19] demonstrating that the composition of inulin and lactulose in the culture medium could stabilize Lactobacillus strains in gastrointestinal conditions, such as bile extract and pancreatic, after 3 hours of incubation. 

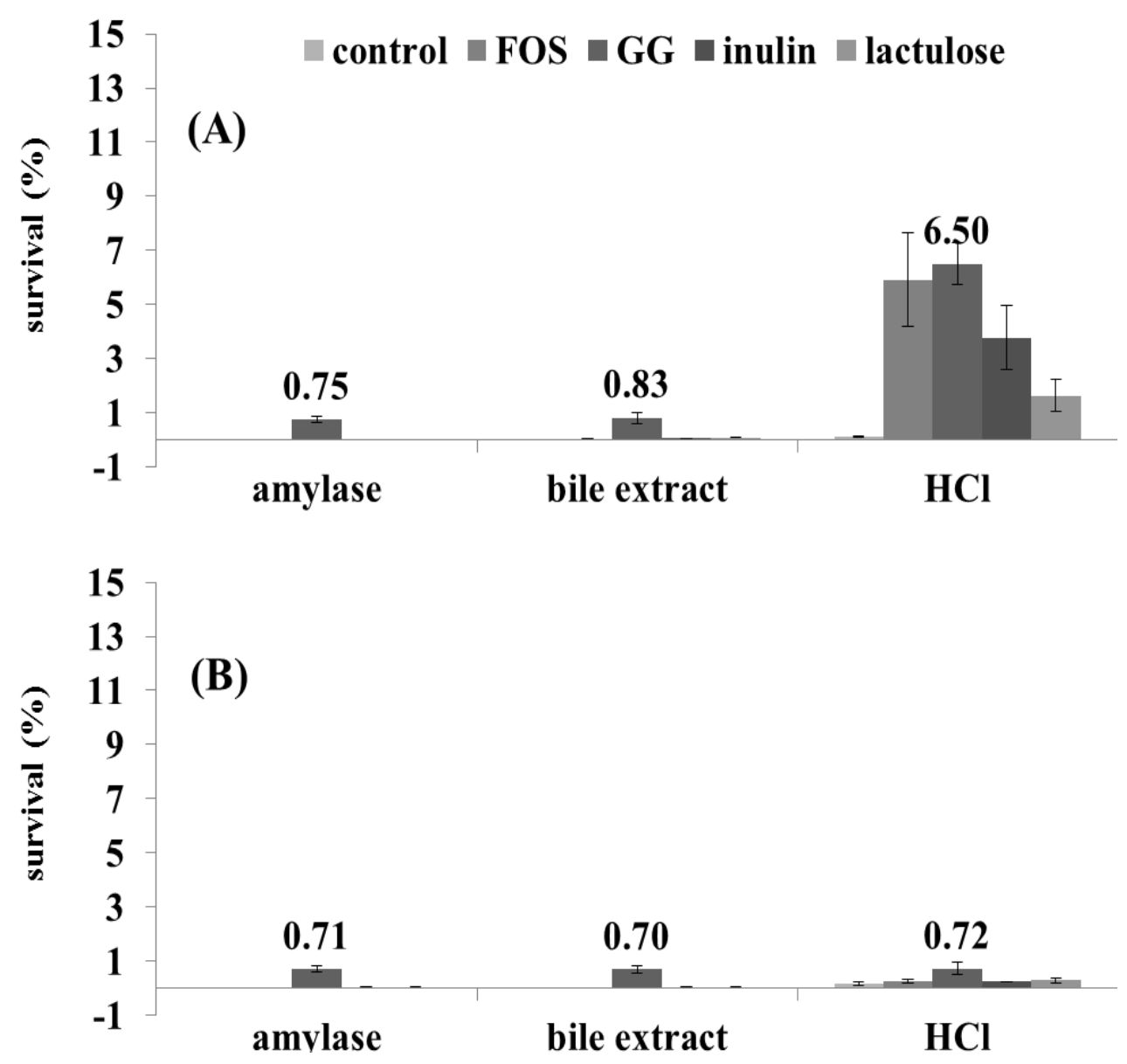

Figure 3. The percentage survival of L. acidophilus (A) and L. plantarum (B) after incubation in gastrointestinal conditions (alpha-amylase, bile extract and $\mathrm{HCl}$ ) for 3 hours in the culture of control, FOS, GG, inulin and lactulose

\section{Lactic acid formation}

Lactic acid concentration from probiotic strains in the culture supplement with FOS, GG, inulin and lactulose after 24 and 48 hours, which is seen in Table 3. From the results, in the culture with FOS and GG displayed higher lactic acid concentration than the others. After 48 hours of incubation, L. plantarum occurred lactic acid concentration in the culture with FOS and GG for 1.25 and $1.22 \mathrm{mg} / \mathrm{ml}$. However, L. acidophilus cultures that contained FOS and GG at the same incubation period performed highest lactic acid concentration for 1.41 and $1.46 \mathrm{mg} / \mathrm{ml}$ (HPLC chromatogram shows in Figure 4). Observable for the culture medium without oligosaccharide (control), lactic acid concentration from both probiotic strains were small amount and not different between 24 and 48 hours. There is evidence from [20] reporting how L. acidophilus ATCC4962 was grown in the medium comprised with FOS, inulin and manitol in order to use these oligosaccharide as a carbon source. After incubation for 20 hours, HPLC chromatogram from the culture with FOS presented higher concentration of some organic acids (lactic acid, formic acid and butyric acid) than the other carbon sources. 
Table 3. Lactic acid concentration from probiotic strains (L. acidophilus and L. plantarum) in the culture medium supplemented with oligosaccharides (FOS, GG, inulin and lactulose) at 24 and 48 hours of cultivation

\begin{tabular}{|ccc|}
\hline \multirow{2}{*}{ Sample } & \multicolumn{2}{c|}{ lactic acid concentration $(\mathbf{m g} / \mathbf{m l})$} \\
\cline { 2 - 3 } & $\mathbf{2 4}$ hours & $\mathbf{4 8}$ hours \\
\hline L. acidophilus & & \\
Control & 0.89 & 0.95 \\
FOS & 0.87 & 1.41 \\
GG & 0.87 & 1.46 \\
inulin & 0.85 & 1.19 \\
lactulose & 0.93 & 1.02 \\
\hline L. plantarum & & \\
Control & 0.66 & 0.81 \\
FOS & 0.65 & 1.25 \\
GG & 0.76 & 1.22 \\
inulin & 0.78 & 0.94 \\
lactulose & 0.75 & 0.98 \\
\hline
\end{tabular}

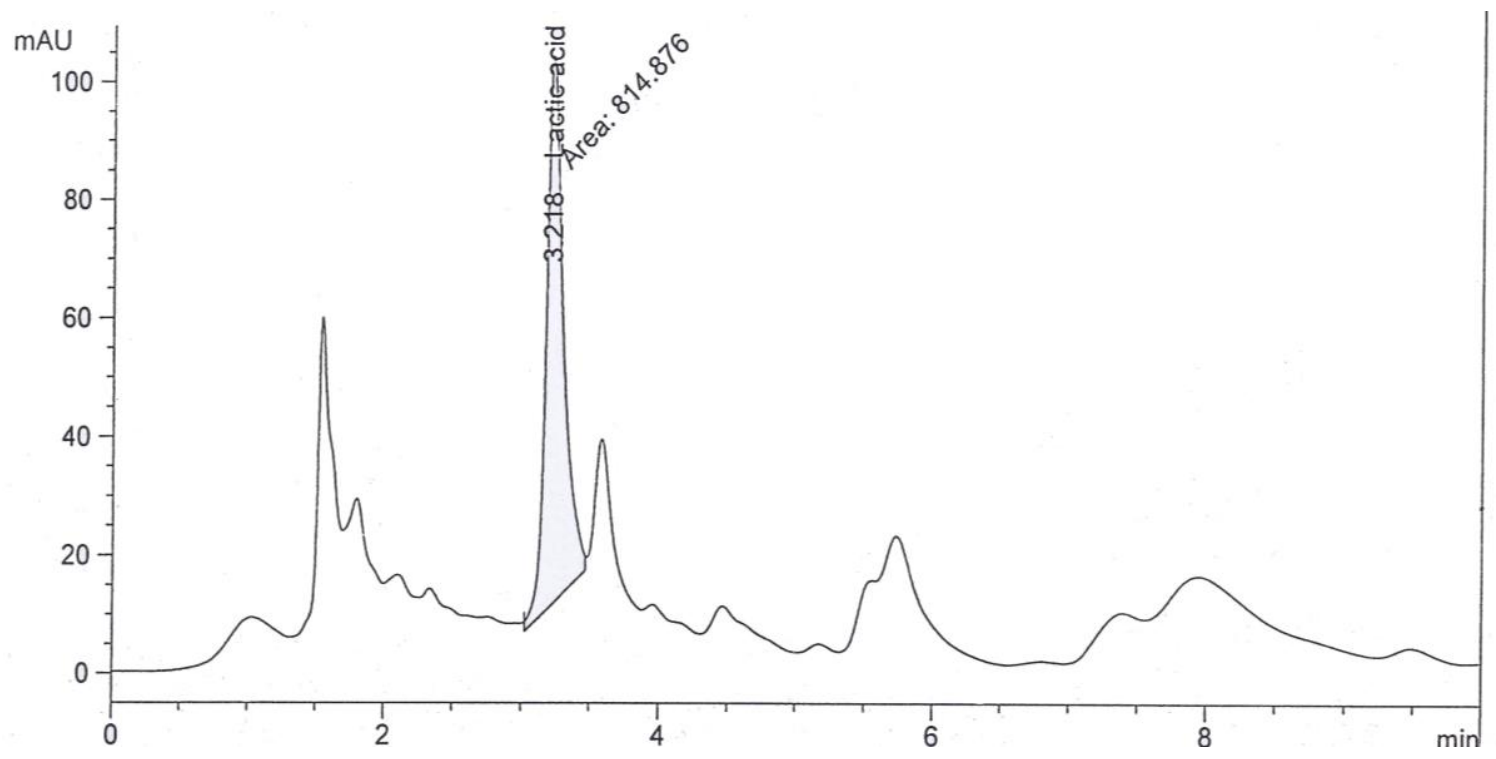

Figure 4. HPLC chromatogram of lactic acid analysis by Dionex Ion-Pac ICE-AS6, $259 \times 9 \mathrm{~mm}$ at $90^{\circ} \mathrm{C}$ from L. acidophilus in the culture with GG at 48 hours of incubation 
CONCLUSION: This study demonstrated the potential of prebiotic properties from glucosylglycerol (GG), which is an organic compound via biocatalytic synthesis, compared to that of three commercial oligosaccharides (FOS, inulin and lactulose). After incubation, the culture with FOS and GG showed high capacity to stimulate both probiotics (L. acidophilus and L. plantarum) and high ability to inhibit 4 intestinal pathogens (B. cereus, E. coli, Samonella paratyphi and Staphyloccous aureus). The culture with GG displayed high tolerance ability of gastrointestinal conditions (alpha amylase, bile extract and $\mathrm{HC}$ ), especially in the culture of $L$. acidophilus, which presented the highest percentage of survival (6.50\%) after being incubated with $\mathrm{HCl}$ for 3 hours. Moreover, lactic acid production from L. acidophilus culture with GG supplemented after 48 hours exhibited maximum concentration of $1.46 \mathrm{mg} / \mathrm{ml}$. From the performance of GG in the prebiotic potential, it could be exploited in application and development to become an efficiency of novel functional food.

Competing Interests: The authors declare that they have no conflicts of interest.

Acknowledgments The author would like to thank Dr. Premsuda Saman (a researcher at Thailand Institute of Scientific and Technological Research), Miss Kanyanee Pimairam and Miss Wanwipa Wansanit (students from the Department of Biotechnology, Ramkhamhaeng University) for the experimental assistance. Financial support from Ramkhamhaeng University is gratefully acknowledged.

\section{REFERENCES:}

1. Li D, Kim JM, Zhengyu J, Zhou J: Prebiotic effectiveness of inulin extracted from edible burdock. Anaerobe 2008, 14: 29-34.

2. Vulevic J, Rastall RA, Gibson GR: Developing a quantitative approach for determining the in vitro prebiotic potential of dietary oligosaccharides. FEMS Microbiol Lett 2004, 236: 153-159.

3. Alejandra ML, Castillo NA, Perdigon G: Anti-infective mechanisms induced by a probiotic Lactobacillus strain against Salmonella enterica serovar Typhimurium infection. Int J Food Microbiol 2010, 138 (3): 223-231.

4. Murry AC, Hinton A, Morrison H: Inhibition of growth of Escherichia coli, Salmonella typhimurium, and Clostridia perfringens on chicken feed media by Lactobacillus salivarius and Lactobacillus plantarum. Int J Poultry Sci 2004, 9(3): 603-607.

5. Holscher HD, Faust KL, Czerkies LA, Litov R, Ziegler EE, Lessin H, Hatch T, Sun S, Tappenden KA: Effects of prebiotic-containing infant formula on gastrointestinal tolerance and fecal microbiota in a randomized controlled trial. JPEN Parenter Enter 2012, 36(suppl 1): 95S-105S.

6. Goedl C, Sawangwan T, Mueller M, Schwarz A, Nidetzky B: A high-yielding biocatalytic process for the production of 2-O-(alpha-D-glucopyranosyl)-sn-glycerol, a natural osmolyte and useful moisturizing ingredient. Angew Chem Int Ed Engl 2008, 47(52): 10086-10089. 
7. Bakker-Zierikzee AM, Alles MS, Knol J, Kok FJ, Tolboom JJ, Bindels JG: Effects of infant formula containing a mixture of galacto- and fructo-oligosaccharides or viable Bifidobacterium animalis on the intestinal microflora during the first 4 months of life. Br J Nutr 2005, 94(5): 783-790.

8. Barile D, Rastall RA: Human milk and related oligosaccharides as prebiotics. Curr Opin Biotechnol 2013, 24(2): 214-219.

9. Kolida S, Tuohy K, Gibson GR: Prebiotic effects of inulin and oligofructose. Br J Nutr 2002, 87(suppl 2): S193-S197.

10. Guerra-Ordaz AA, Gonzalez-Ortiz G, Ragione RM, Woodward MJ, Collins JW, Perez JF, Martin-Orue SM: Lactulose and Lactobacillus plantarum, a potential complementary synbiotic to control postweaning colibacillosis in piglets. Appl Environ Microbiol 2014, 80(16): 4879-4886.

11. Siragusa S, Di Cagno R, Ercolini D, Minervini F, Gobbetti M, De Angelis M: Taxonomic structure and monitoring of the dominant population of lactic acid bacteria during wheat flour sourdough type I propagation using Lactobacillus sanfranciscensis starters. Appl Environ Microb 2009, 75(4): 1099-1109.

12. Rousseau V, Lepargneur JP, Roques C, Remaud-Simeon M, Paul, F: Prebiotic effects of oligosaccharides on selected vaginal lactobacilli and pathogenic microorganisms. Anaerobe 2005, 11(3): 145-153.

13. Kondepudi KK, Ambalam P, Nilsson I, Wadström T, Ljungh Å: Prebiotic-nondigestible oligosaccharides preference of probiotic bifidobacteria and antimicrobial activity against Clostridium difficile. Anaerobe 2012, 18(5): 489-497.

14. Mullin WJ, Emmons DB: Determination of organic acids and sugars in cheese, milk and whey by high performance liquid chromatography. Food Res Int 1997, 30(2): 147-151.

15. Huebner J, Wehling RL, Hutkins RW: Functional activity of commercial prebiotics. Int Dairy J 2007, 17(7): 770-775.

16. Ignatova-Ivanova, T, Mehmedova S, Ermenlieva N, Ivanov R: Growth characteristics of Lactobacillii strains cultivated on media with fructooligosaccharides and their antimicrobial activity. J BioSci Biotech 2012, SE/ONLINE: 169-172.

17. Sawangwan T, Goedl C, Nidetzky B: Glucosylglycerol and glucosylglycerate as enzyme stabilizers. Biotechnol J 2010, 5(2): 187-191.

18. Hernandez-Hernandez O., Muthaiyan A, Moreno FJ, Montilla A, Sanz ML, Ricke SC: Effect of prebiotic carbohydrates on the growth and tolerance of Lactobacillus. Food Microbiol 2012, 30(2): 355-361.

19. Nazzaro F, Florinda F, Barbara N, Annarita P, Pierangelo O: The prebiotic source influences the growth, biochemical features and survival under simulated gastrointestinal conditions of the probiotic Lactobacillus acidophilus. Anaerobe 2012, 18(3): 280-285.

20. Liong MT, Shah NP: Production of organic acids from fermentation of mannitol, fructo-oligosaccharide and inulin by a cholesterol removing Lactobacillus acidophilus strain. J Appl Microbiol 2005, 99(4): 783-793. 